\title{
Corrigendum: The seco-iridoid pathway from Catharanthus roseus
}

Karel Miettinen, Lemeng Dong, Nicolas Navrot, Thomas Schneider, Vincent Burlat, Jacob Pollier, Lotte Woittiez, Sander van der Krol, Raphaël Lugan, Tina Ilc, Robert Verpoorte, Kirsi-Marja Oksman-Caldentey, Enrico Martinoia, Harro Bouwmeester, Alain Goossens, Johan Memelink \& Danièle Werck-Reichhart

Nature Communications 5:3606 doi: 10.1038/ncomms4606 (2014); Published 7 Apr 2014; Updated 24 Jun 2014

While this Article was undergoing peer review, Salim et al. (2013), Asada et al. (2013) and Salim et al. (2014) published individual steps of the (seco)iridoid biosynthesis pathway in Catharanthus roseus, including the molecular and biochemical characterization of 7-deoxyloganic acid hydroxylase, 7-deoxyloganetic acid glucosyl transferase and 7-deoxyloganetic acid synthase, which are in agreement with our results. These papers should have been cited at the end of the Discussion section.

Salim, V., Yu, F., Altarejos, J. \& De Luca, V. Virus induced gene silencing identifies Catharanthus roseus 7-deoxyloganic acid 7-hydroxylase, a step in iridoid and monoterpenoid indole alkaloid biosynthesis. Plant J. 76, 754-765 (2013).

Asada, K. et al. A 7-deoxyloganetic acid glucosyltransferase contributes a key step in secologanin biosynthesis in Madagascar periwinkle. Plant Cell 25, 4123-4134 (2013).

Salim, V., Wiens, B., Masada-Atsumi, S., Yu, F. \& De Luca, V. 7-Deoxyloganetic acid synthase catalyzes a key 3 step oxidation to form 7-deoxyloganetic acid in Catharanthus roseus iridoid biosynthesis. Phytochemistry 101, 23-31 (2014). 